\title{
Research on PDMA communication system based on complete complementary sequence
}

\author{
Shufeng Li $i^{*}$, Baoxin Su and Libiao Jin
}

\author{
*Correspondence: shufeng_2004@ \\ 163.com \\ School of Information and \\ Communication Engineering, State \\ Key Laboratory of Media \\ Convergence and Communication, \\ Communication University of China, \\ |Beijing 100024, People's Republic of \\ China
}

\begin{abstract}
Pattern division multiple access (PDMA) is a new non-orthogonal multiple access (NOMA) technology. It is proposed to meet the challenge of $5 \mathrm{G}$ large-scale connectivity and high-frequency spectral efficiency. Compared with traditional orthogonal multiple access (OMA), PDMA can support more users through the allocation of non-orthogonal resources. Due to perfect aperiodic correlation, complete complementary sequence (CCS) greatly improves the spectrum efficiency of the system. It has been widely used in wireless communication and radar sensing, and it still has research value in 5G. In order to apply the advantages of CCS to NOMA communication system, this paper proposes a system model of CCS spread spectrum coding based on PDMA. CCS is used as spread spectrum code to improve the performance of PDMA communication system. At the same time, on the basis of spread spectrum technology, this paper analyzes the average power allocation algorithm and water-filling power allocation algorithm, and a dynamic power allocation algorithm based on the transmission rate and practical application is proposed. The simulation results show that the system model can effectively improve the performance of the system.
\end{abstract}

Keywords: Non-orthogonal multiple access, Pattern division multiple access, Complete complementary sequence, Spread spectrum coding, Power allocation, Dynamic

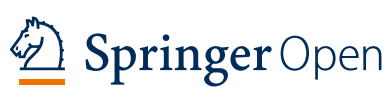

\section{Introduction}

The rapid development of mobile communication and Internet of things poses challenges to the fifth generation (5G) wireless communication system [1-4]. As a research hotspot in recent years, it is generally believed that non-orthogonal multiple access (NOMA) is a promising multiple access technology for 5G and beyond cellular networks [5]. As one of the key technologies of 5G network, NOMA can improve the spectrum efficiency through time-frequency resource multiplexing and large-scale connected user grouping [3]. In NOMA communication system, the allocation strategy of power, subcarrier, and other resources are also the focus of research. By comparing the system performance of traditional orthogonal multiple access (OMA) and NOMA, we can see that NOMA has outstanding advantages such as improving spectrum efficiency

(c) The Author(s). 2020 Open Access This article is licensed under a Creative Commons Attribution 4.0 International License, which permits use, sharing, adaptation, distribution and reproduction in any medium or format, as long as you give appropriate credit to the original author(s) and the source, provide a link to the Creative Commons licence, and indicate if changes were made. The images or other third party material in this article are included in the article's Creative Commons licence, unless indicated otherwise in a credit line to the material. If material is not included in the article's Creative Commons licence and your intended use is not permitted by statutory regulation or exceeds the permitted use, you will need to obtain permission directly from the copyright holder. To view a copy of this licence, visit http://creativecommons.org/licenses/by/4.0/. 
and cell edge throughput, large-scale connection, and reducing transmission delay and signaling cost [6].

Pattern division multiple access (PDMA) is a new NOMA technology based on the early research of successive interference cancelation amenable multiple access $[7,8]$. PDMA distinguishes multiple user signals at the transmitter through the use of space domain, power domain, and code domain alone or jointly, and at the same time, multiple users share the same time-frequency resources. It uses successive interference cancelation (SIC) detection algorithm or belief propagation (BP) detection algorithm to carry out multi-user detection at the receiver, so as to achieve the optimal overall performance of the communication system [9]. The sparsity of PDMA resource mapping matrix provides favorable conditions for the receiver to use the $\mathrm{BP}$ algorithm to detect multi-user data. The use of multiple signal domains enables PDMA to meet 5G large connection and high speed. In [10], the design of the PDMA transmitter and receiver is introduced in detail, and the basic performance evaluation of the PDMA uplink and downlink is given. In [8], the design criteria of PDMA pattern matrix in MMTC and eMBB deployment scenarios are introduced, and the performance of PDMA pattern matrix in different dimensions and row mediums is analyzed. The technical requirements of the application scenarios facing the mobile Internet of things PDMA technology are summarized in [11].

Golay introduced the concept of complementary pair in the study of infrared spectroscopy [12]. He proposed a pair of binary sequences whose aperiodic auto-correlation sum becomes zero except for zero shift, and it is called complementary pair. After Golay's work, Turyn and Taki et al. studied the properties of complementary pairs and their relations with other types of sequences, extending Golay's idea to a complementary set $[13,14]$. Complete complementary sequence (CCS) is a generalization of Golay complementary code, which is a set of sequences with ideal correlation sum. CCS is widely used because of their perfect aperiodic correlation [15]. Complementary pairs can be applied in many fields, such as radar sensing, channel estimation, and 3G standard synchronization. In [16], recursive algorithms for generating polyphase and multilevel complementary sequences are given. In recent years, some complementary set structures have been proposed by solving equations [17], recursive construction [18], and discrete Fourier transform (DFT) matrix [19]. In addition, the application of CCS is also extended to the fields of image processing [20] and signal processing. Under the condition of the same signal-to-noise ratio (SNR), the spread spectrum communication gains stronger anti-noise interference performance [21, 22]. In this paper, CCS spread spectrum is applied to PDMA system as an enhancement technology, which can broaden the bandwidth and reduce the effect of block error rate (BLER). At the same time, compared with the traditional $\mathrm{m}$-sequence and Gold sequence spread spectrum, the BLER of CCS is simulated and analyzed.

In NOMA system, the channel gain difference between users can be converted into multiplexing gain by superposition coding. Therefore, power allocation has a great impact on system performance [23]. At present, the more classic power allocation algorithms are mainly divided into three categories: equal power allocation, joint power allocation, and step-by-step power allocation. Equal power allocation is the simplest one, as long as the total power is divided equally in each subchannel; the core algorithm of joint and distributed is water-filling algorithm [24]. The existing research on 
power allocation in NOMA mainly considers the quality of service (QoS) and fairness criteria of users. Two user and multi-user QoS power allocation schemes are proposed in [25] and [26]. In [27], a proportional fair (PF) power allocation scheme for two users under different criteria is proposed. And in [28], the optimal power allocation scheme is also proposed from the perspective of PF.

In this paper, a communication system based on PDMA with CCS spread spectrum and power allocation is studied, through the analysis of CCS, average power allocation, water-filling power allocation, and dynamic power allocation. And the simulation results are compared and analyzed.

The main contents of this paper are as follows:

Section 2 introduces the proposed method. In Section 3, we introduce the downlink CCS spread spectrum communication and power allocation system based on PDMA. Section 4 introduces the knowledge of CCS. In Section 5, a dynamic power allocation algorithm based on transmission rate is proposed. Section 6 analyzes the simulation results in many cases.

\section{Methods}

Due to the joint use of multiple signal domains, PDMA has unique conditions in meeting 5G connection and high speed. CCS is widely used in wireless communication and radar sensing because of its good aperiodic correlation. In practical application, the resolution of different types of data is different, and the required transmission rate is also different. In order to reduce the waste of power, based on the existing water-filling power allocation, this paper proposes a dynamic power allocation with transmission rate as the index to save resources and improve the system performance.

This paper presents a system model of CCS spread spectrum and dynamic power allocation based on PDMA. In order to verify the effectiveness of the algorithm, we have conducted a variety of experiments to obtain comparison results. First of all, we take $\mathrm{m}$-sequence and Gold sequence as examples and compare them with CCS in different dimensional pattern matrices. Experiments show that the spread spectrum performance of CCS is the best, and the longer the sequence, the better the performance. Then, we use $2 \times 3$ pattern matrix to do average power allocation, water-filling power allocation, and dynamic power allocation respectively on the basis of three kinds of sequence spread spectrum, which proves that the dynamic power allocation algorithm has certain advantages. The specific analysis can be found in Section 6 .

\section{System model}

The downlink technical framework of PDMA is shown in Fig. 1. PDMA uses characteristic pattern to distinguish users at the transmitter and serial interference cancelation algorithm with low complexity and high performance to realize multi-user detection at the receiver. In Fig. 1, user 1 and user 2 are in one multi-antenna beam transmission direction, while user 3 and user 4 are in the other beam transmission direction. For users in the same direction, the time-frequency domain PDMA pattern can be used to distinguish users in the same direction to realize non-orthogonal transmission. The time-frequency domain PDMA pattern combined with space domain resource multiplexing can simultaneously transmit downlink data for 4 users. 


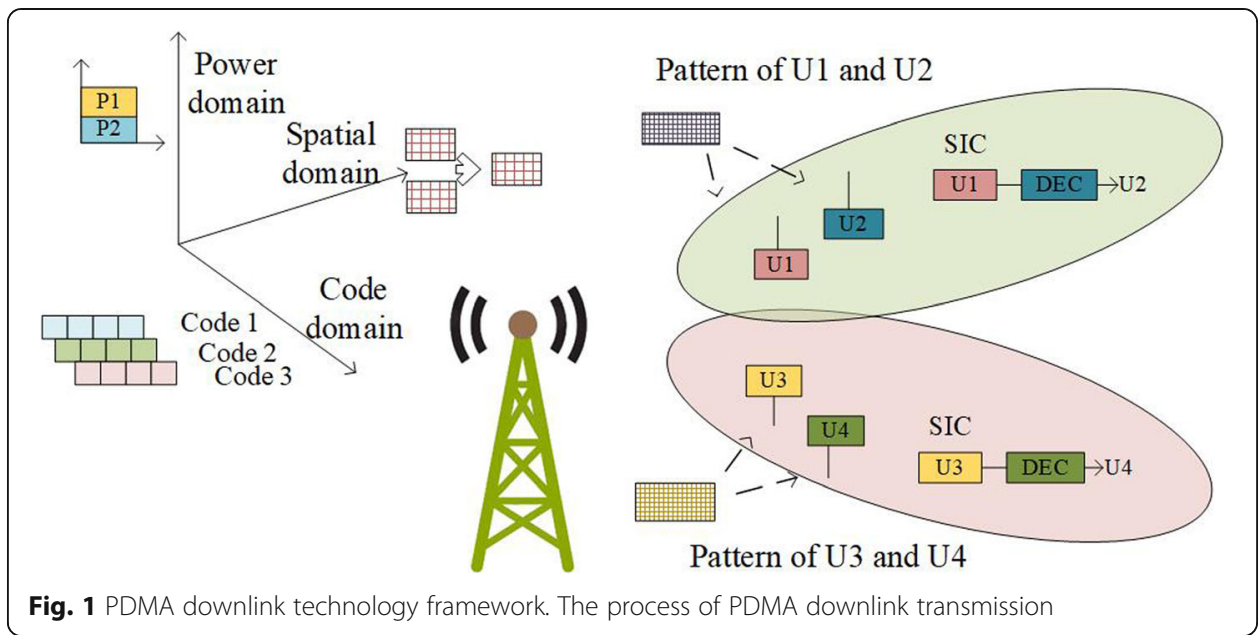

At the receiver, the message passing algorithm (MPA) and SIC can be selected to eliminate the interference. When SIC detection algorithm is used, different users are in different detection layers, and their equivalent diversity is different. According to the detection order of the receiver, the first detected user has the lowest equivalent receiving diversity, and the last detected user has the highest. Therefore, we need to introduce inconsistent transmission diversity degree to ensure that all users can achieve as consistent and high equivalent diversity degree as possible after detection, so as to improve the detection performance.

In this paper, we propose a PDMA based downlink CCS spread spectrum communication and power allocation system, as shown in Fig. 2. Firstly, the modulated transmission signal is spread through the corresponding spread spectrum sequence. In order to combine the advantages of CCS into NOMA communication system, CCS is used as spread spectrum code to improve the implementation of non-orthogonal multiple access communication and improve the transmission performance of the system. And the introduction of fast Fourier transform (FFT) greatly reduces the complexity of system implementation.

Suppose that $J$ user equipment (UE) of PDMA system are mapped to $K$ resource elements $(\mathrm{RE})$ in time and frequency domain, and each UE has a unique PDMA mode. The received signal $y$ can be expressed as:

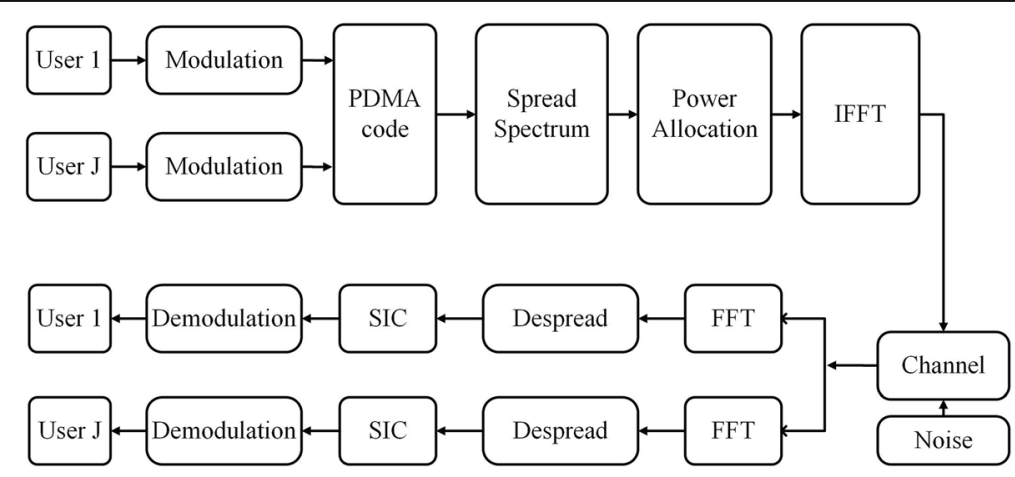

Fig. 2 System model. The spread spectrum and power allocation system model based on PDMA 


$$
y_{k}=\operatorname{diag}\left(h_{k}\right) \mathbf{H}_{\mathrm{PDMA}} \tilde{x}+\tilde{n_{k}}
$$

where

$$
\tilde{x}=\sum_{i=1}^{K} \sqrt{P_{i}} x_{i}
$$

$\mathbf{H}$ represents the equivalent channel response matrix composed of PDMA multi-user coding matrix from the sender to the receiver and the real wireless channel response matrix, $\mathbf{H}_{\text {PDMA }}$ is the PDMA pattern matrix with dimension $K \times J, \boldsymbol{h}_{k}$ is the wireless channel response of user k. $S$ is the spread spectrum sequence with length L, $\tilde{x}$ represents the transmitted signal vector after power allocation, and $\tilde{\mathbf{n}}$ is Gaussian noise with dimension $K \times J$.

The definition of CCS $\left\{A_{k}, B_{k}\right\}$ in Fig. 3 is a CCS set satisfying complete orthogonality; $f_{c}$ is the carrier frequency. The sequence length is $L$, which is expressed as follows:

$$
\left\{\begin{array}{l}
A_{k}=\left(a_{k}^{1}, a_{k}^{2}, \ldots, a_{k}^{L}\right) \\
B_{k}=\left(b_{k}^{1}, b_{k}^{2}, \ldots, b_{k}^{L}\right)
\end{array}\right.
$$

The baseband signal of the $k$ th user after spread spectrum can be represented as

$$
X_{k}=x_{k} S_{k}
$$

where $x_{k}$ is the original user information data, $S_{k}$ is the spread spectrum sequence, which can be expressed as

$$
\begin{gathered}
S_{k}(t)=A_{k}(t)+B_{k}\left(t-\tau_{a b}\right) \\
=\sum_{l=1}^{L}\left[a_{k, i}^{l} \operatorname{rect}_{c}\left(\frac{t-l T_{c}}{T_{d}}\right)+b_{k, i}^{l} \operatorname{rect}_{c}\left(\frac{t-\tau_{a b}-l T_{c}}{T_{d}}\right)\right]
\end{gathered}
$$

where $T_{d}$ is the pulse period, $T_{c}$ is the chip period, $\tau_{a, b}$ represents the time delay from $a_{k}$ to $b_{k}$, and rect $t_{c}(t)$ represents the rectangular window function

$$
\operatorname{rect}_{c}(t)=\left\{\begin{array}{rr}
1 & 0 \leq t \leq T_{c} \\
0 & \text { else }
\end{array}\right.
$$

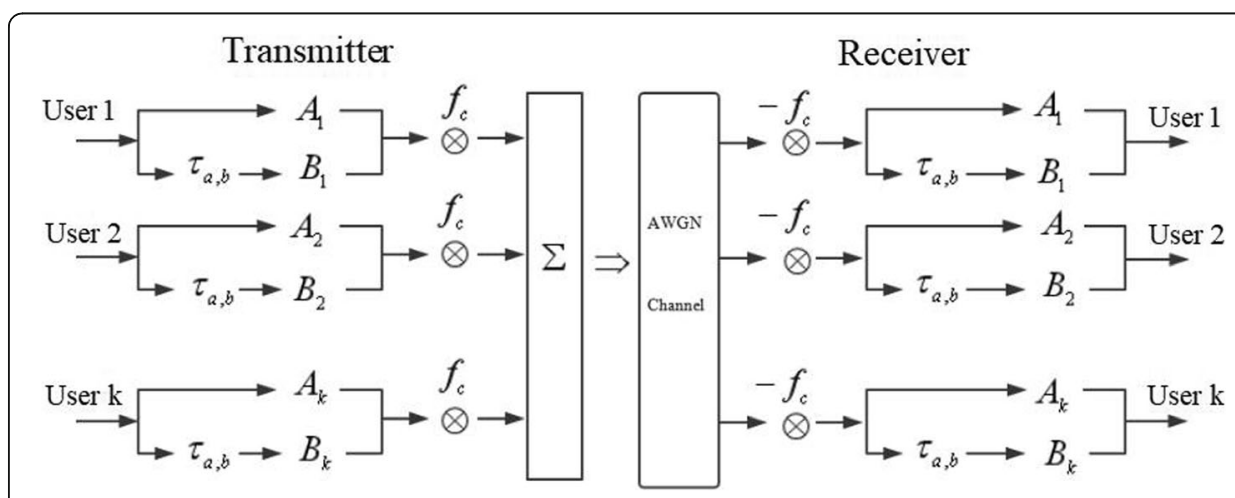

Fig. 3 Structure diagram of spread spectrum system simulation model based on complete complementary sequence pair. The structure of spread spectrum system based on complete complementary sequence pairs 


\section{Complete complementary sequence (CCS)}

\subsection{Complementary sequence pair and complementary set}

For any nonzero time shift, the sum of the aperiodic auto-correlation function (AACF) of the complementary pair is zero. The idea of Golay is extended to mutually orthogonal complementary code (MOCC) [29], where the sum of aperiodic cross-correlation function (ACCF) is zero for any two complementary set in any time shift.

For any two sequences $A=\left\{a_{0}, a_{1}, \cdots, a_{L-1}\right\}$ and $B=\left\{b_{0}, b_{1}, \cdots, b_{L-1}\right\}$ of length $L$, their aperiodic correlation functions at time shift $t$ are defined as

$$
\theta_{A, B}(\tau)=\left\{\begin{array}{c}
\sum_{k=0}^{L-1-\tau} A[k] B^{*}[k+\tau], 0 \leq \tau \leq L-1 \\
\sum_{k=0}^{L-1+\tau} A[k-\tau] B^{*}[k], 1-L \leq \tau \leq 0 \\
0 \quad \text { else }
\end{array}\right.
$$

where ()" is complex conjugate. When $A \neq B$, the above formula is called ACCF, when $A=B$, it is called AACF. AACF of $A$ is written as $\theta_{A}(\tau)$.

The Z-transform of sequence $A$ is

$$
A(Z)=\sum_{k=0}^{L-1} A[k] Z^{-k}
$$

The complex conjugate of the above formula is expressed as follows:

$$
A^{*}(Z)=\sum_{k=0}^{L-1} A^{*}[k] Z^{-k}
$$

Let $A^{R}[k]=A[L-1-k]$ denote the reversion sequence, which means that the elements are inverted in time, and its $Z$-transform is expressed as

$$
A^{R}(Z)=Z^{-L+1} A\left(Z^{-1}\right)
$$

The $Z$-transform of ACCF is

$$
\theta_{A, B}(Z)=\sum_{k=0}^{L-1} \theta_{A, B}[k] Z^{-k}=A\left(Z^{-1}\right) B^{*}(Z)
$$

Similarly, we can get the $Z$-transform $\theta_{A}(Z)$ of AACF.

Two binary sequences $A=\left\{a_{0}, a_{1}, \cdots, a_{L-1}\right\}$ and $B=\left\{b_{0}, b_{1}, \cdots, b_{L-1}\right\}$ of length $L$ are known, if their AACF satisfy

$$
\theta_{A}(Z)+\theta_{B}(Z)=c
$$

and $c$ is a positive real number, $A$ and $B$ are called a pair of complementary sequence pair.

A set of sequences $\left\{A^{0}(Z), A^{1}(Z), \cdots A^{K-1}(Z)\right\}$, if they satisfy (13), it is called complementary set.

$$
\sum_{i=0}^{K-1} \theta_{A}(k)=\sum_{i=0}^{K-1} A^{(i)}\left(Z^{-1}\right) A^{(i) *}(Z)=c
$$

Let a set of Kcolumn vectors $\left\{A_{0}(Z), A_{1}(Z), \cdots, A_{K-1}(Z)\right\}$, the size of each vector is M, 


$$
A_{\mu}(Z)=\left[A_{\mu}^{(0)}(Z), A_{\mu}^{(1)}(Z), \cdots, A_{\mu}^{(M-1)}(Z)\right]^{T}
$$

where $0 \leq \mu \leq K-1, A_{\mu}^{(m)}(Z)$ is a sequence, $m \in\{0,1, \cdots, M-1\}$.

The $Z$-transform $(0 \leq \mu, v \leq K-1)$ of the sum of ACCF of $A_{\mu}(Z)$ and $A_{v}(Z)$ is defined as

$$
S_{A_{\mu}, A_{\nu}}(Z)=\sum_{r=0}^{M-1} \theta_{A_{\mu}^{(r)}, A_{\nu}^{(r)}}(Z)=\tilde{A}_{\nu}\left(Z^{-1}\right) A_{\mu}\left(Z^{-1}\right)
$$

where $\tilde{A}_{v}\left(Z^{-1}\right)=A_{v}^{H}(Z), H$ is Hermitian operation.

If a $(k, m, l)$ - mutually orthogonal complementary code set (MOCCS) $D$ satisfies

$$
S_{A_{\mu}, A_{\nu}}(Z)=D \delta(\mu-v)
$$

it has been proved that $k \leq m$, when $k=m, D$ is a group of CCS, and $\delta$ is the Kronecker increment function.

\subsection{Recursive construction of complete complementary sequences (CCS)}

Complete complementary sequences (CCS), which is composed of mutually orthogonal complementary sequences, is a set of complementary sequences with ideal autocorrelation and cross-correlation properties. For example, given $A=\left\{a_{0}, a_{1}, \cdots, a_{L-1}\right\}$ and $B=\left\{b_{0}, b_{1}, \cdots, b_{L-1}\right\}$ as an initial complementary sequence pair seed, the length of each seed is $L$, and $a_{i}, b_{i} \in(-1,1,-j, j)$ is a four phase sequence, the auto-correlation expression of $A_{0}$ and $B_{0}$ satisfies

$$
\theta_{A_{0}, A_{0}}(\tau)+\theta_{A_{0}, A_{0}}(\tau)=\left\{\begin{array}{cc}
2 L & \tau=0 \\
0 & \tau \neq 0
\end{array}\right.
$$

The recursive construction process is shown in the Fig. 4, where $\left\{A_{1}\right\}=\left\{A_{0}, B_{0}\right\}$, $\left\{B_{1}\right\}=\left\{A_{0},-B_{0}\right\}$,in the same way $\left\{A_{1}^{\prime}\right\}=\left\{-\overline{B_{0}^{*}}, \overline{A_{0}^{*}}\right\},\left\{B_{1}^{\prime}\right\}=\left\{-\overline{B_{0}^{*}},-\overline{A_{0}^{*}}\right\},\left\{A_{1}, B_{1}\right\}$, and $\left\{A_{1}^{\prime}, B_{1}^{\prime}\right\}$ is a pair of complete complementary sequence sets, where $A_{0}^{*}$ is the conjugate sequence of $A_{0}$ and $\overline{A_{0}^{*}}$ is the reversion sequence of $A_{0}^{*}$.

The seed sequences $A_{0}=\{1, j,-j,-1, j\}$ and $B_{0}=\{1,1,1, j,-j\}$ with a given length of 5 , a set of CCS with length 10 can be obtained.

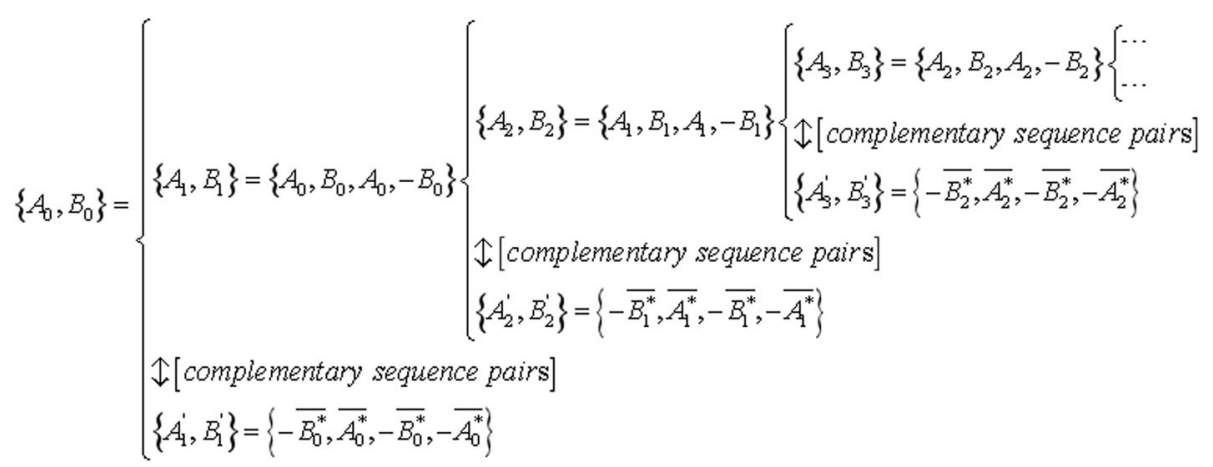

Fig. 4 Recursive construction algorithm flow chart. A recursive algorithm for constructing complete complementary sequences 


$$
\begin{aligned}
& A_{1}=\left\{A_{0}, \quad B_{0}\right\}=\{1, j,-j,-1, j, 1,1,1, j,-j\} \\
& B_{1}=\left\{A_{0},-B_{0}\right\}=\{1, j,-j,-1, j,-1,-1,-1,-j, j\} \\
& A_{1}^{\prime}=\left\{-\overline{B_{0}^{*}}, \overline{A_{0}^{*}}\right\}=\{-j, j,-1,-1,-1,-j,-1, j,-j, 1\} \\
& B_{1}^{\prime}=\left\{-\overline{B_{0}^{*}},-\overline{A_{0}^{*}}\right\}=\{-j, j,-1,-1,-1, j, 1,-j, j,-1\}
\end{aligned}
$$

Similarly, CCS of length 20 and 40 can be obtained by iteration. By deriving the fuzzy function of complete complementary sequence, the complete orthogonality of complete complementary sequence is proved [30].

Suppose that $K$ resource elements transmit signals, the CCS with length $L$ can be spread by formula (5) to obtain the spread spectrum sequence $S_{k}$ with the size of $K \times L$, at this time, the received signal $\tilde{y}_{k}$ can be expressed as:

$$
\tilde{y}_{k}=\mathbf{H} x S_{k}+\tilde{\mathbf{n}}_{\mathbf{k}}
$$

At the receiving end, the received signal is obtained by despreading the corresponding spread spectrum code.

\section{Power allocation}

Power allocation means that under certain conditions, the capacity of the system can reach the ideal value by designing the transmitted signal [31]. Downlink power allocation is to allocate the power among multiple users considering the fairness of users, so as to reduce the interference between users and improve the spectrum efficiency of the system. The signal after power allocation can be expressed as:

$$
x=\sqrt{P_{1}} x_{1}+\sqrt{P_{2}} x_{2}+\cdots+\sqrt{P_{2}} x_{2}=\sum_{i=1}^{K} \sqrt{P_{i}} x_{i}
$$

where $P_{i}$ is the power allocated to the $i$ th user, and $x_{i}$ is the signal vector transmitted. Base station can control the throughput of the system by flexibly controlling the transmission power ratio of users, which is of great help to the improvement of system capacity.

\subsection{Average power allocation}

Average power allocation is to distribute the transmit power to each channel equally.

For average power allocation, the expression of channel capacity is

$$
C=E\left\{\log _{2}\left[\operatorname{det}\left(I_{N r}+\frac{\mathbf{H} P \mathbf{H}^{H}}{\sigma^{2}}\right)\right]\right\}
$$

where $E\{\}$ is to find mathematical expectation, det means to find the determinant of a square matrix, $I_{N r}=P=\operatorname{diag}\left(\sqrt{P_{1}}, \sqrt{P_{2}}, \cdots, \sqrt{P_{N}}\right),()^{H}$ is the complex conjugate transposition, and $\mathrm{His}$ the channel matrix. Therefore, the channel capacity can be simplified as

$$
C=\frac{1}{N} \sum_{n=1}^{N}\left\{\log _{2}\left(I_{N r}+\frac{\mathbf{H H}^{H}}{\sigma^{2}}\right)\right\}
$$




\subsection{Water-filling power allocation}

The water-filling algorithm allocates the transmit power adaptively according to the channel condition. It usually allocates more power when the channel condition is good, and allocates less power when it is bad, so as to maximize the transmission rate. When the power satisfies $P=\sum_{i=1}^{K} P_{i}$, the channel capacity can be obtained from Shannon's theorem,

$$
C=\sum_{i=1}^{K} \log _{2}\left(1+\frac{P_{i} \lambda_{i}}{\sigma^{2}}\right)
$$

where $K$ is the number of independent subchannels. In order to maximize $C$, Lagrange multiplier method is used to introduce the function

$$
Z=\sum_{n=1}^{K} \log _{2}\left(1+\frac{P_{i} \lambda_{i}}{\sigma^{2}}\right)+L\left(P-\sum_{i=1}^{K} P_{i}\right)
$$

Let $\frac{\partial Z}{\partial P_{i}}=0$,

$$
\frac{\partial Z}{\partial P_{i}}=\frac{\lambda_{i} / \sigma^{2}}{\ln 2\left(1+P_{i} \lambda_{i} / \sigma^{2}\right)}-L=0
$$

we can get

$$
P_{i}=\frac{1}{\ln 2 \cdot L}-\frac{\sigma^{2}}{\lambda_{i}}=\mu-\frac{\sigma^{2}}{\lambda_{i}}
$$

where $\mu$ is a constant, which represents the water-filling plane, $P_{i}=\left(\mu-\frac{\sigma^{2}}{\lambda_{i}}\right)^{+}$can be obtained by extrapolation, and $(\alpha)^{+}$is $\max (\alpha, 0), \lambda_{i}$ is the $i$ th eigenvalue of the channel matrix, and $\sigma^{2}$ is the noise variance.

\section{Algorithm: Water-filling power allocation algorithm}

step1. Let the total power be $P$ and the number of iterations $r=1$, according to $\lambda=\operatorname{SVD}(H)$,

$$
\text { calculate } \mu=\frac{1}{K-r+1}\left(P+\sum_{i=1}^{K-r+1} \frac{\sigma^{2}}{\lambda_{i}}\right)
$$

step2. The power distributed on each antenna is $P_{i}=\mu-\frac{\sigma^{2}}{\lambda_{i}}$

step3. If the energy channel allocated to the minimum gain is negative, then $P_{K-r+1}=0$, $r=r+1$, jump to step 1 ; if any $P_{i}$ is not negative, the optimal water-filling power allocation strategy can be obtained

\subsection{Dynamic power allocation}

In practical applications, when transmitting different types of data, such as highdefinition video, ordinary video, pictures, and text, the resolution is different, and the required transmission rate is also different. If the same type of power is distributed, a lot of power will be wasted. Therefore, based on the existing water-filling power allocation, this paper proposes a dynamic power allocation based on transmission rate. By 
Table 1 PDMA pattern matrix. The pattern matrix of different dimensions selected for simulation

\begin{tabular}{|c|c|c|c|c|c|c|c|c|}
\hline \multirow{2}{*}{$\begin{array}{l}\text { Matrix dimension and overload rate }(\boldsymbol{a}) \\
\text { Dimension } 2 \times 3 \\
a=150 \%\end{array}$} & \multicolumn{8}{|c|}{ Pattern matrix } \\
\hline & & $\begin{array}{l}1 \\
0\end{array}$ & & & & & & \\
\hline $\begin{array}{l}\text { Dimension } 3 \times 6 \\
a=200 \%\end{array}$ & {$\left[\begin{array}{l}1 \\
0 \\
1\end{array}\right.$} & $\begin{array}{l}0 \\
1 \\
0\end{array}$ & $\begin{array}{l}0 \\
1 \\
1\end{array}$ & $\begin{array}{l}1 \\
0 \\
0\end{array}$ & 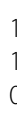 & 0 & & \\
\hline $\begin{array}{l}\text { Dimension } 4 \times 6 \\
a=150 \%\end{array}$ & {$\left[\begin{array}{l}1 \\
1 \\
1 \\
1\end{array}\right.$} & $\begin{array}{l}1 \\
1 \\
0\end{array}$ & $\begin{array}{l}0 \\
1 \\
1 \\
0\end{array}$ & $\begin{array}{l}0 \\
0 \\
1 \\
1\end{array}$ & c & 0 & & \\
\hline $\begin{array}{l}\text { Dimension } 4 \times 8 \\
a=200 \%\end{array}$ & {$\left[\begin{array}{l}1 \\
1 \\
1 \\
1\end{array}\right.$} & $\begin{array}{l}1 \\
1 \\
0 \\
0\end{array}$ & $\begin{array}{l}0 \\
1 \\
1 \\
0\end{array}$ & $\begin{array}{l}0 \\
0 \\
1 \\
1\end{array}$ & c & 1 & & $\begin{array}{l}0 \\
0\end{array}$ \\
\hline
\end{tabular}

changing the water-filling plane, the resource is saved and the system performance is improved.

It can be seen from eq. (26) that

$$
\mu=\frac{1}{\ln 2 \cdot L}
$$

By introducing the scale factor $\beta_{i}=\frac{r_{i}}{K}, r_{i}$ represents the rate of the $i$ th transmission

$$
\sum_{l=1}^{K} r_{l}
$$

information, we can get

$$
\tilde{\mu}_{i}=\mu \beta_{i}=\frac{\beta_{i}}{\ln 2 \cdot L}
$$

so,

$$
P_{i}=\frac{\beta_{i}}{\ln 2 \cdot L}-\frac{\sigma^{2}}{\lambda_{i}}=\tilde{\mu}_{i}-\frac{\sigma^{2}}{\lambda_{i}}
$$

where $\tilde{\mu}_{i}$ is a constant, which is dynamic water-filling plane, we can get $P_{i}=\left(\tilde{\mu}_{i}-\frac{\sigma^{2}}{\lambda_{i}}\right)^{+}$ $\beta_{i}$ is the scaling factor of transmission rate, $\lambda_{i}$ is the $i$ th eigenvalue of channel matrix, and $\sigma^{2}$ is noise variance.

\section{Simulation results}

In this section, the system model is simulated.

Table 2 Different order feedback coefficients of m-sequence. The m-sequence feedback coefficients of different orders selected for simulation

\begin{tabular}{ll}
\hline Order & Feedback coefficient \\
\hline 3 & {$\left[\begin{array}{llllll}1 & 0 & 1\end{array}\right]$} \\
4 & {$\left[\begin{array}{lllll}1 & 0 & 0 & 1\end{array}\right]$} \\
5 & {$\left[\begin{array}{llllll}1 & 0 & 0 & 1 & 1\end{array}\right]$} \\
6 & {$\left[\begin{array}{llllll}1 & 0 & 0 & 0 & 0 & 1\end{array}\right]$} \\
\hline
\end{tabular}


Table 3 Optimal pairs of Gold sequences with different orders. The Gold sequence optimization pairs of different orders selected in the simulation

\begin{tabular}{|c|c|c|c|c|c|}
\hline \multirow{2}{*}{$\frac{\text { Order }}{4}$} & \multicolumn{5}{|c|}{ Preferred pair } \\
\hline & {$\left[\begin{array}{ll}1 & 0 \\
0 & 0\end{array}\right.$} & $\begin{array}{l}0 \\
1\end{array}$ & $\begin{array}{l}1] \\
1]\end{array}$ & & \\
\hline 5 & {$\left[\begin{array}{ll}1 & 0 \\
1 & 1\end{array}\right.$} & $\begin{array}{l}1 \\
1\end{array}$ & $\begin{array}{c}0 \\
1\end{array}$ & $\begin{array}{l}1 \\
1\end{array}$ & \\
\hline 6 & {$\left[\begin{array}{ll}1 & 0 \\
{[1} & 1\end{array}\right.$} & $\begin{array}{l}0 \\
0\end{array}$ & $\begin{array}{l}0 \\
0\end{array}$ & $\begin{array}{l}0 \\
1\end{array}$ & $\begin{array}{c}1] \\
1]\end{array}$ \\
\hline
\end{tabular}

Firstly, we analyze the performance of CCS spread spectrum based on PDMA, and based on PDMA, we simulated other common spread spectrum sequences, such as $\mathrm{m}$ sequence and Gold sequence [32].

The CCS selects the seed sequence $A_{0}=\{1, j,-j,-1, j\}$ and $B_{0}=\{1,1,1, j,-j\}$ with the length of 5 and obtains the spread spectrum sequence with length of 10, 20, and 40 respectively by iterative method. Table 1 shows the selected pattern matrix with different dimensions and overload rate, Table 2 shows the feedback coefficients of different dimensions of the selected $\mathrm{m}$-sequence, and Table 3 shows the corresponding $\mathrm{m}$ sequence optimization pairs for producing Gold sequences of different orders.

\subsection{Performance comparison and analysis of three spread spectrum sequences under different pattern matrices}

Figures 5, 6 and 7 show the simulation comparison of SNR to BLER with CCS, msequence, and Gold sequence with different spreading order or different spreading code length under four different pattern matrices. BLER is a statistical parameter of bad data block received on the channel in unit time, which is used to measure the system
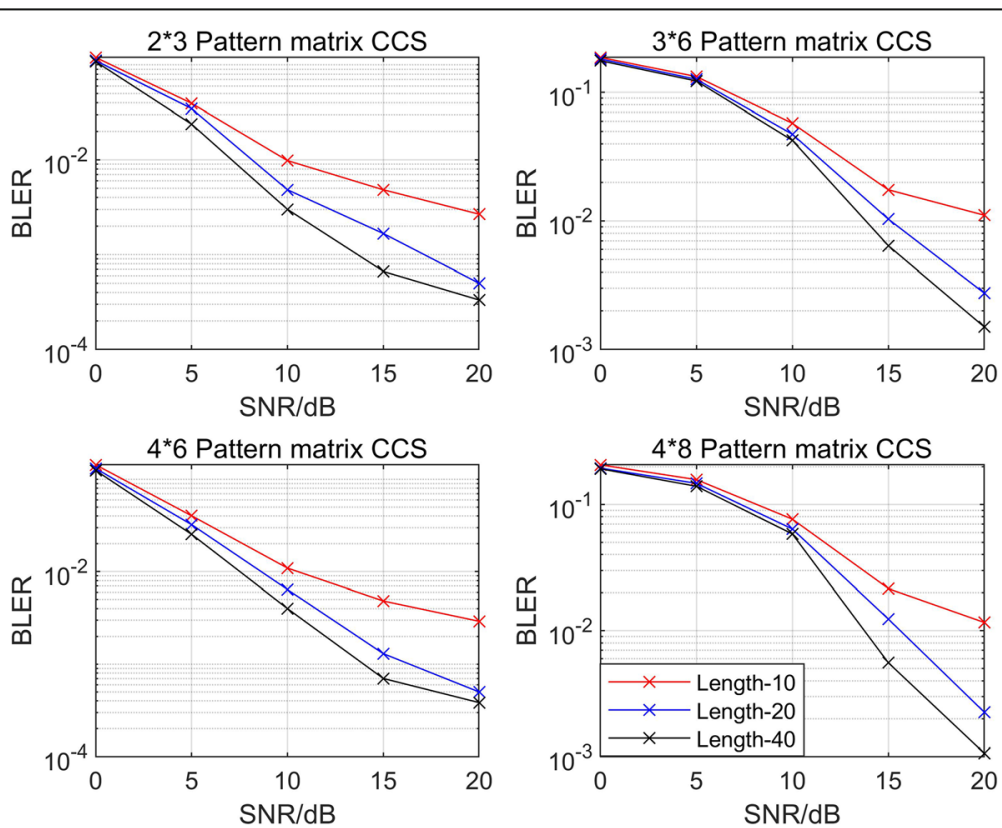

Fig. 5 CCS spread spectrum based on PDMA pattern matrix with different dimensions. The spread spectrum performance comparison of CCS with length of 10,20, and 40 under different dimensional pattern matrices 

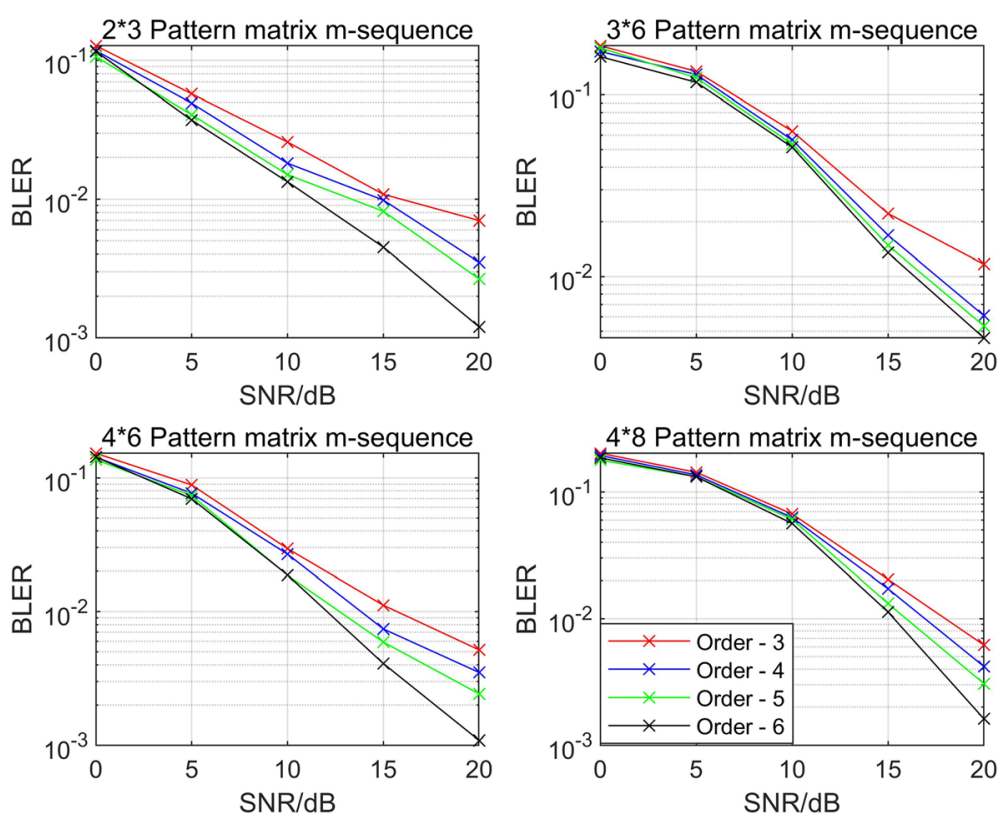

Fig. 6 m-sequence spread spectrum based on PDMA pattern matrix with different dimensions. The spread spectrum performance comparison of m-sequence of order 3 , order 4 , order 5 , and order 6 under different dimensional pattern matrices. The dimension of pattern matrix is $2 \times 3$
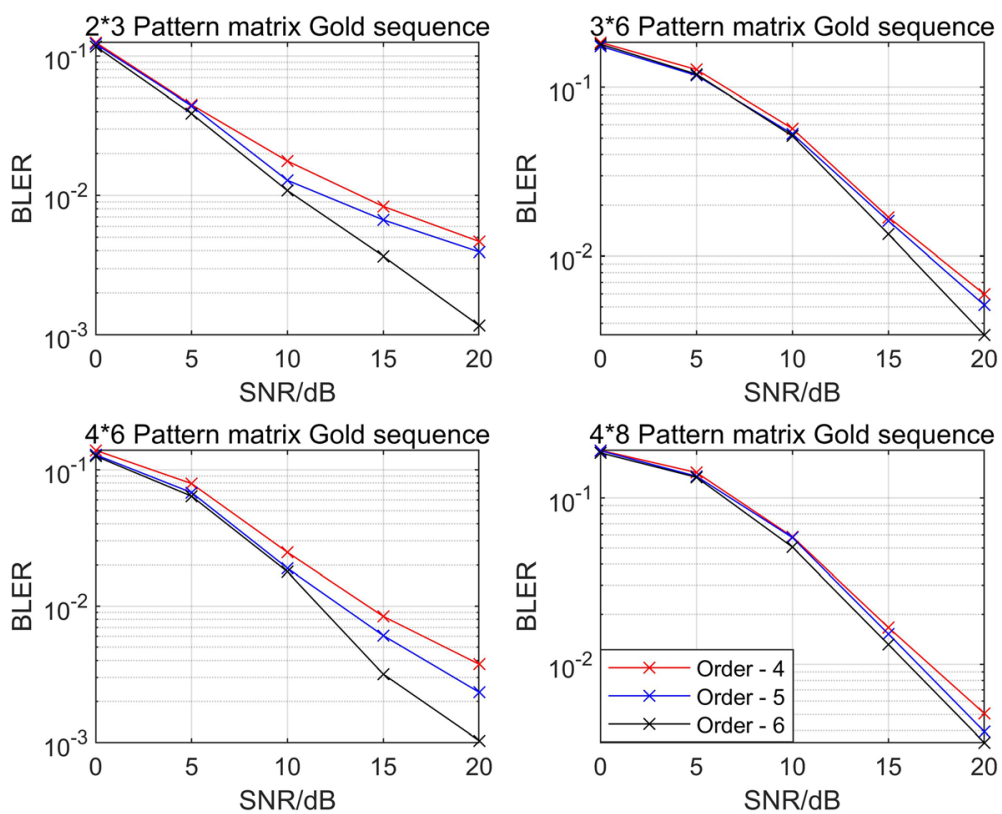

Fig. 7 Gold sequence spread spectrum based on PDMA pattern matrix with different dimensions. The spread spectrum performance comparison of Gold sequence of order 4, order 5, and order 6 under different dimensional pattern matrices. The dimension of pattern matrix is $2 \times 3$ 

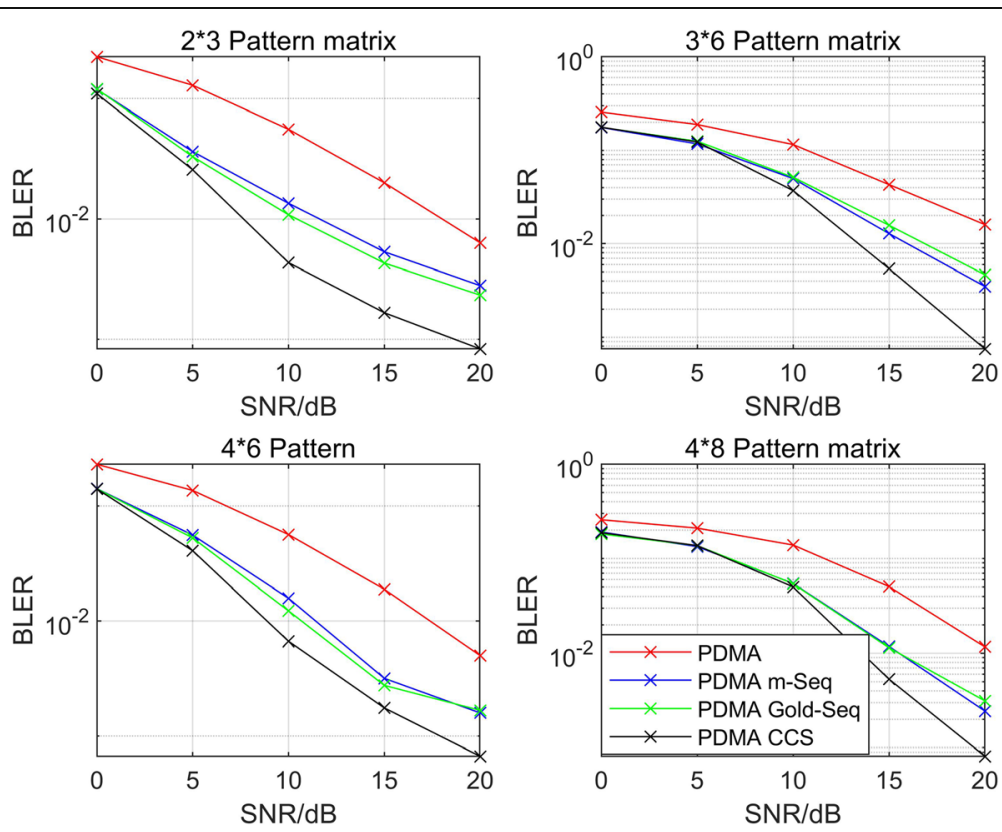

Fig. 8 Three spread spectrum sequences based on PDMA pattern matrix with different dimensions. The performance comparison of three spread spectrum sequences with different dimension pattern matrix. The m-sequence and Gold sequence adopt 6-order feedback coefficient, and the code length of CCS is 40 . The dimension of pattern matrix is $2 \times 3$

performance. The selection of pattern matrix and feedback coefficient of each sequence is shown in Tables 1,2 and 3.

As can be seen in Figs. 5, 6 and 7, for the pattern matrix with different dimensions, the change trend of BLER is the same when the spread spectrum order and the length of spread spectrum code are increasing. Therefore, for PDMA spread spectrum, BLER decreases with the increase of feedback order and code length.

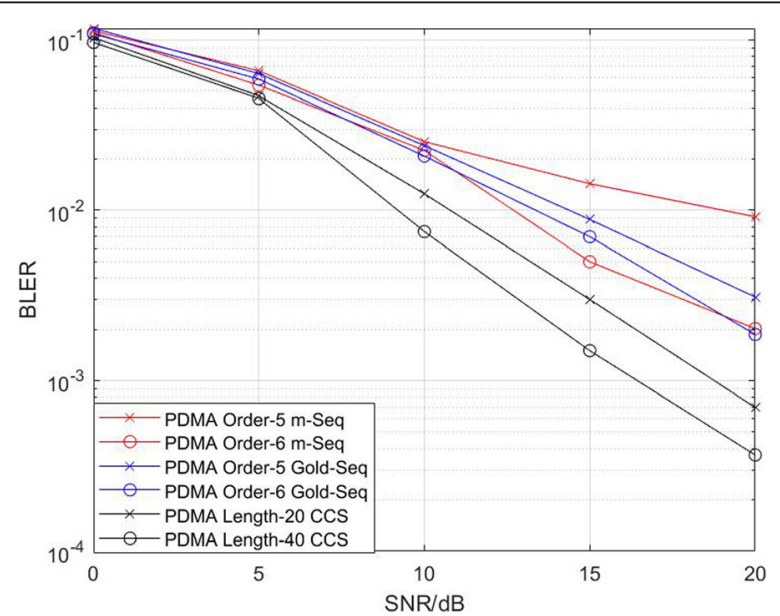

Fig. 9 Performance comparison of three spread spectrum sequences under average power allocation. The comparison of average power allocation performance of 5-order 6-order m-sequence, 5-order 6-order Gold sequence, and CCS with length of 20 and 40. The dimension of pattern matrix is $2 \times 3$ 


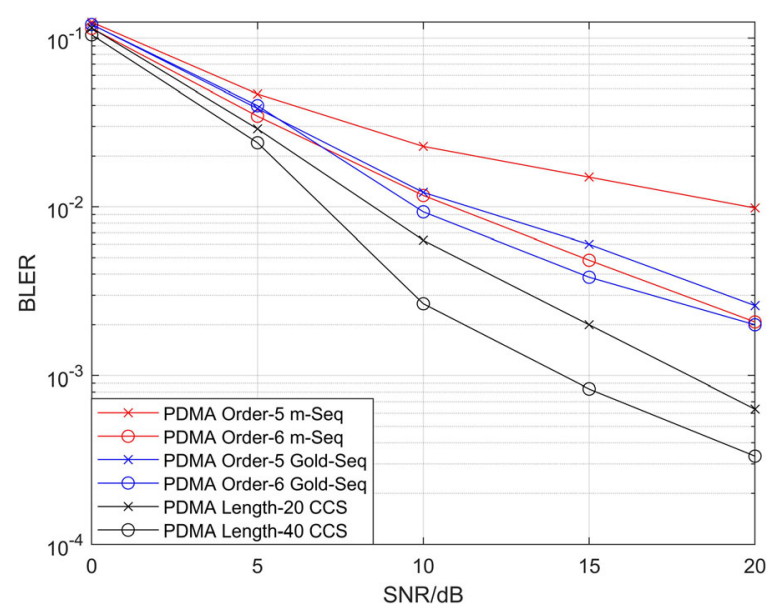

Fig. 10 Performance comparison of three spread spectrum sequences under water-filling power allocation. The comparison of water-filling power allocation performance of 5-order 6-order m-sequence, 5-order 6order Gold sequence, and CCS with length of 20 and 40. The dimension of pattern matrix is $2 \times 3$

For different pattern matrices, when the overload rate is the same, the change trend of BLER with SNR is roughly the same. And under the same SNR, through data comparison, we can see that the BLER of a matrix with a higher dimension will be slightly smaller, but the gap is very small.

Figure 8 shows the performance comparison of three spread spectrum sequences with different dimension pattern matrix. m-sequence and Gold sequence adopt 6-order feedback coefficient, and the code length of CCS is 40.

It can be seen that the three kinds of spread spectrum sequences can improve the system performance, and the performance of the CCS is the best. Because the autocorrelation property of $\mathrm{m}$-sequence is better than that of Gold sequence, the system performance of m-sequence is slightly better than that of Gold sequence in lowdimensional pattern matrix. With the increase of matrix dimension, the performance gap between $\mathrm{m}$-sequence and Gold sequence gradually decreases.

\subsection{Performance comparison of different spread spectrum sequences under several power allocation algorithms}

It can be seen in Figs. 5, 6, 7 and 8 that the general trend of BLER is the same under different dimensional pattern matrices. Therefore, in order to reduce the computational complexity, we take the pattern matrix of $2 \times 3$ dimension as an example for analysis.

Figures 9 and 10 show the comparison of SNR and BLER of three spread spectrum sequences under the condition of average power allocation and water-filling power allocation. It shows that with the increase of SNR, the longer the spread spectrum code is, and the smaller the BLER is. Under the average power allocation and water-filling

Table 4 Common video and image resolution and optimal transmission rate. The resolutions of several common videos and images and their corresponding optimal transmission rate

\begin{tabular}{lllllll}
\hline Resolution & 480P & 720P & 1080P & QCIF & CIF & 4CIF \\
\hline Rate/(kbps) & 1800 & 3500 & 8500 & 128 & 384 & 1500 \\
\hline
\end{tabular}




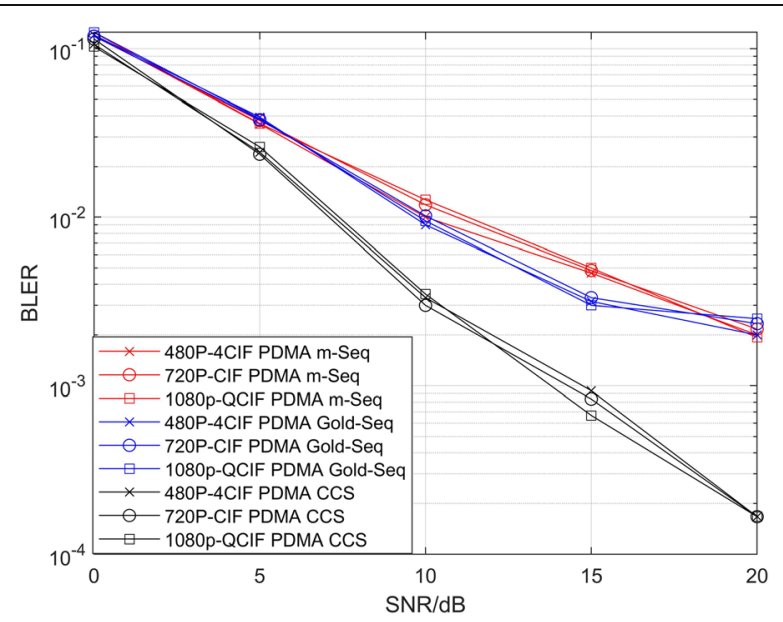

Fig. 11 Dynamic power allocation at different transmission data. The performance comparison of the three spread spectrum sequences under the condition of dynamic power allocation transmitting different kinds of information at the same time. The dimension of pattern matrix is $2 \times 3$

power allocation, the spread spectrum performance of CCS is better than that of $\mathrm{m}$ sequence and Gold sequence.

Table 4 shows the resolutions of several common videos and images and their corresponding optimal transmission rate. Figure 11 shows the performance comparison of the three spread spectrum sequences under the condition of dynamic power allocation transmitting different kinds of information at the same time. Similarly, $2 \times 3$ pattern matrix is selected, the spread spectrum order of m-sequence and Gold sequence is 6, and the length of spread spectrum code of CCS is 40 .

As can be seen in the Fig. 11, the impact of the rate difference multiple on the system performance is basically the same, so this method is feasible.

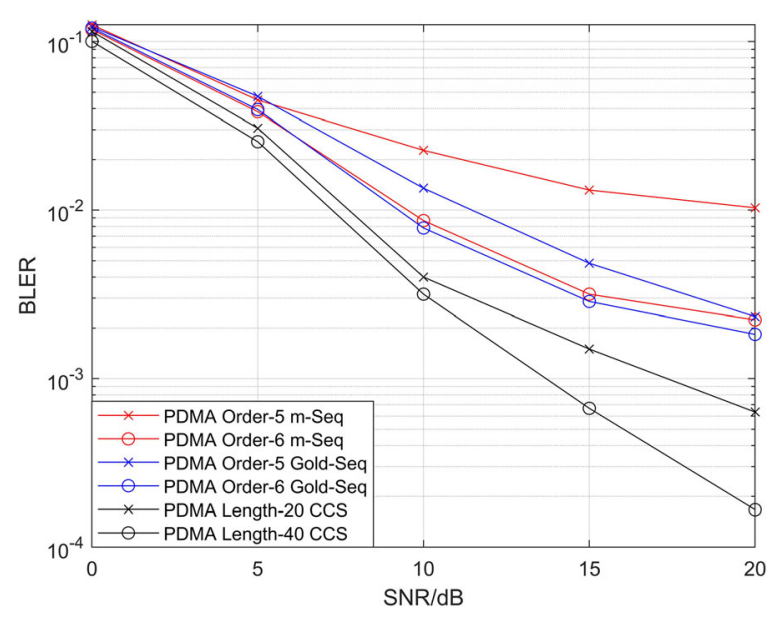

Fig. 12 Performance comparison of three spread spectrum sequences under dynamic power allocation. The comparison of dynamic power allocation performance of 5-order 6-order m-sequence, 5 -order 6-order Gold sequence, and CCS with length of 20 and 40 . The dimension of pattern matrix is $2 \times 3$ 
Figure 12 shows the simulation comparison of SNR and BLER of CCS, msequence, and Gold sequence under dynamic power allocation. The simulation is carried out by transmitting video with resolution of 1080P and image of QCIF at the same time.

It can be seen that with the increase of SNR, the order of spread spectrum sequence is higher, the length of spread spectrum code is longer, and the BLER is smaller.

Figure 13 shows the SNR and BLER comparison of average power allocation, waterfilling power allocation, and dynamic power allocation algorithms under CCS, msequence, and Gold sequence respectively. Taking $2 \times 3$ pattern matrix as an example, 6-order m-sequence and Gold sequence spread spectrum code are selected, the length of fully CCS is 40 , and 1080P and QCIF are selected for dynamic transmission data.

We can see that the performance of dynamic power allocation is slightly better than that of water-filling power allocation. And the best effect is obtained when the CCS spread spectrum and dynamic power allocation are used.

\section{Conclusions}

This paper mainly studies the communication system performance of CCS spread spectrum based on PDMA. Firstly, we introduce the concept of CCS and iterative construction method. Then, the average power allocation algorithm and the water-filling power allocation algorithm are analyzed, and we propose a dynamic power allocation algorithm based on transmission rate from the perspective of practical application. In the simulation of the system model, we compare the spread spectrum performance of $\mathrm{m}$-sequence and Gold sequence with CCS. At the same time, based on these three spread spectrum sequences, three power allocation algorithms are used respectively. The simulation results show that BLER is much smaller than m-sequence and Gold sequence when CCS is used as spread spectrum code. Dynamic power allocation can further improve system performance based on spread spectrum technology.

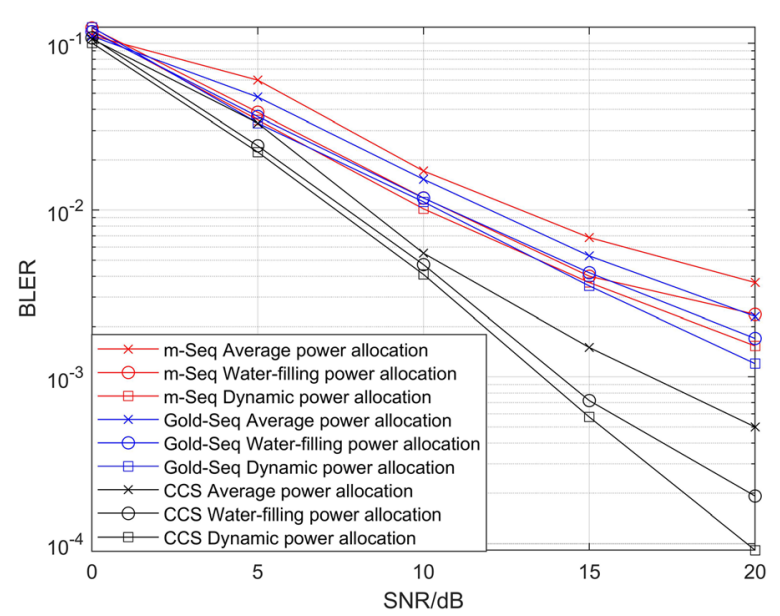

Fig. 13 Performance comparison of several power allocation algorithms under three spreading sequences. The performance comparison of three power allocation under three spread spectrum sequences. The dimension of pattern matrix is $2 \times 3$ 


\section{Abbreviations}

PDMA: Pattern division multiple access; NOMA: Non-orthogonal multiple access; OMA: Orthogonal multiple access; CCS: Complete complementary sequence; SIC: Successive interference cancelation; BP: Belief propagation; DFT: Discrete Fourier transform; BLER: Block error rate; QoS: Quality of service; PF: Proportional fair; MPA: Message passing algorithm; FFT: Fast Fourier transform; UE: User equipment; RE: Resource elements; AACF: Aperiodic autocorrelation function; ACCF: Aperiodic cross-correlation function; MOCC: Mutually orthogonal complementary code

\section{Acknowledgements}

The authors wish to acknowledge Dr. Hongda Wu, University of York University, Canada, for his help in writing this paper.

\section{Authors' contributions}

Conceptualization, Shufeng Li; methodology, Baoxin Su; supervision, Libiao Jin; writing-review and editing, Shufeng Li. The authors read and approved the final manuscript.

\section{Funding}

This work was supported by National Nature Science Funding of China (NSFC): 61401407 and the Fundamental Research Funds for the Central Universities.

\section{Availability of data and materials}

Not applicable

\section{Competing interests}

The authors declare that they have no competing interests.

Received: 17 August 2020 Accepted: 29 September 2020

Published online: 14 October 2020

\section{References}

1. L. Chettri, R. Bera, A comprehensive survey on internet of things (IoT) toward $5 \mathrm{G}$ wireless systems. IEEE Internet Things J. $7(1), 16-32(2020)$

2. L. He, Q. Guo, J. Zhong, X. Wang, M. Li, Y. Teng, 5 G network performance analysis and verification based on ubiquitous electricity Internet of Things (2020 IEEE 4th Information Technology, Networking, Electronic and Automation Control Conference (ITNEC), Chongqing, 2020), pp. 2613-2617

3. A. Li, Y. Lan, X. Chen, H. Jiang, Non-orthogonal multiple access (NOMA) for future downlink radio access of 5G. China Comm. 12(Supplement), 28-37 (2015)

4. B. Wang, K. Wang, Z. Lu, T. Xie, J. Quan, Comparison study of non-orthogonal multiple access schemes for $5 \mathrm{G}$ (2015 IEEE International Symposium on Broadband Multimedia Systems and Broadcasting, Ghent, 2015), pp. 1-5

5. A.A. Khansa, Y. Yin, G. Gui, H. Sari, Power-Domain NOMA or NOMA-2000? (2019 25th Asia-Pacific Conference on Communications (APCC), Ho Chi Minh City, 2019), pp. 336-341

6. K. Lu, Z. Wu, X. Shao, "A survey of non-orthogonal multiple access for 5G," 2017 IEEE 86th Vehicular Technology Conference (VTC-Fall, Toronto, ON, 2017), pp. 1-5

7. J. Zeng, B. Li, X. Su, L. Rong, R. Xing, Pattern division multiple access (PDMA) for cellular future radio access (2015 International Conference on Wireless Communications \& Signal Processing (WCSP), Nanjing, 2015), pp. 1-5

8. B. Ren, Y. Wang, X. Dai, K. Niu, W. Tang, Pattern matrix design of PDMA for $5 G$ UL applications. China Comm. 13(Supplement2), 159-173 (2016)

9. B. Ren et al., Advanced IDD receiver for PDMA uplink system (2016 IEEE/CIC International Conference on Communications in China (ICCC), Chengdu, 2016), pp. 1-6

10. S. Chen, B. Ren, Q. Gao, S. Kang, S. Sun, K. Niu, Pattern division multiple access-a novel nonorthogonal multiple access for fifth-generation radio networks. IEEE Trans. Veh. Technol. 66(4), 3185-3196 (2017)

11. C. Wang, PDMA generator design and power allocation optimization [D] (University of Electronic Science and technology, 2019)

12. M. Golay, Complementary series. IRE Transac. Inform. Theory 7(2), 82-87 (1961)

13. R. Turyn, Ambiguity functions of complementary sequences (Corresp.). IEEE Trans. Inf. Theory 9(1), 46-47 (1963)

14. Y. Taki, H. Miyakawa, M. Hatori, S. Namba, Even-shift orthogonal sequences. IEEE Trans. Inf. Theory 15(2), 295-300 (1969)

15. C.-C. Tseng, C. Liu, Complementary sets of sequences. IEEE Trans. Inf. Theory 18(5), 644-652 (1972)

16. S.Z. Budisin, New complementary pairs of sequences. Electron. Lett. 26(13), 881-883 (1990)

17. X. Yang, Y. Mo, D. Li, M. Bian, New complete complementary codes and their analysis (IEEE GLOBECOM 2007 - IEEE Global Telecommunications Conference, Washington, DC, 2007), pp. 3899-3904

18. R. Appuswamy, A.K. Chaturvedi, A new framework for constructing mutually orthogonal complementary sets and ZCZ sequences. IEEE Trans. Inf. Theory 52(8), 3817-3826 (2006)

19. Y. Jin, H. Koga, Basic properties of the complete complementary codes using the DFT matrices and the Kronecker products (2008 International Symposium on Information Theory and Its Applications, Auckland, 2008), pp. 1-6

20. S.E. El-Khamy, M.A. Mokhtar, N.O. El-Ganainy, New techniques for image change and motion detection based on complete complementary code arrays (Proceedings of the Twenty-First National Radio Science Conference, 2004. NRSC 2004, Cairo, 2004), p. C35-1

21. Z. Yuchi, L. Shufeng, J. Libiao, Application of MIMO enhancement technique based on spread spectrum method (2016 IEEE International Conference on Electronic Information and Communication Technology (ICEICT), Harbin, 2016), pp. 317-320

22. Z. Liupiao, Research on the construction and sequence allocation of spread spectrum sequences [D] (Xi'an University of Electronic Science and technology, 2018)

23. H. Wang, R. Zhang, R. Song, S. Leung, A novel power minimization precoding scheme for MIMO-NOMA uplink systems. IEEE Commun. Lett. 22(5), 1106-1109 (2018) 
24. L. Zhiwei, F. Chunyan, Z. Tiankui, Z. Jieying, Power allocation algorithm for multi cell interference coordination in OFDMA systems [J]. J Beijing Univ. Posts Telecomm. 01, 71-74 (2008)

25. M.M. El-Sayed, A.S. Ibrahim, M.M. Khairy, Power allocation strategies for non-orthogonal multiple access (2016 International Conference on Selected Topics in Mobile \& Wireless Networking (MoWNeT), Cairo, 2016), pp. 1-6

26. Z. Yang, W. Xu, C. Pan, Y. Pan, M. Chen, On the optimality of power allocation for NOMA downlinks with individual QoS constraints. IEEE Commun. Lett. 21(7), 1649-1652 (2017)

27. J. Choi, Power allocation for max-sum rate and max-min rate proportional fairness in NOMA. IEEE Commun. Lett. 20(10), 2055-2058 (2016)

28. F. Liu, P. Mähönen, M. Petrova, Proportional fairness-based power allocation and user set selection for downlink NOMA systems (2016 IEEE International Conference on Communications (ICC), Kuala Lumpur, 2016), pp. 1-6

29. S.Z. Budisin, New multilevel complementary pairs of sequences. Electron. Lett. 26(22), 1861-1863 (1990)

30. S. Li, L. Zhang, J. Chen, Y. Zhou, Application of complete complementary sequence in MIMO radar [J]. J Beijing Univ. Aeronautics Astronautics 36(05), 564-569 (2010)

31. G. Zhenggang, Channel capacity analysis of MIMO system [J]. J Ningbo Polytechnic 14(02), 26-31 (2010)

32. W. Haihong, Comparison and application of $m$-sequence and gold sequence in CDMA spread spectrum communication [J]. J Kashi Normal Univ. 31(03), 39-43 (2010)

\section{Publisher's Note}

Springer Nature remains neutral with regard to jurisdictional claims in published maps and institutional affiliations.

Submit your manuscript to a SpringerOpen ${ }^{\circ}$ journal and benefit from:

- Convenient online submission

$\checkmark$ Rigorous peer review

- Open access: articles freely available online

- High visibility within the field

- Retaining the copyright to your article

Submit your next manuscript at $\boldsymbol{\nabla}$ springeropen.com 